\title{
TRANSFINITE DIAMETER WITH GENERALIZED POLYNOMIAL DEGREE
}

\author{
SIONE MA'U
}

\begin{abstract}
We prove a Chebyshev transform formula for a notion of (weighted) transfinite diameter that is defined using a generalized notion of polynomial degree. We also generalize Leja points to this setting. As an application of our main formula, we prove that in the unweighted case, these generalized Leja points recover the transfinite diameter.
\end{abstract}

\section{INTRODUCTION}

The usual grading of polynomials by (total) degree may be described geometrically. Let

$$
\Sigma:=\left\{\left(x_{1}, \ldots, x_{N}\right) \in \mathbb{R}_{+}^{N}: x_{1}, \ldots, x_{N} \geq 0, x_{1}+\cdots+x_{N} \leq 1\right\}
$$

be the standard $n$-dimensional unit simplex in $\mathbb{R}_{+}^{N}$. Then $\operatorname{deg}(p) \leq n$ may be reformulated as $p \in \operatorname{Poly}(n \Sigma)$, where

$$
\operatorname{Poly}(n \Sigma):=\left\{p(z)=\sum_{J \in n \Sigma \cap \mathbb{Z}_{+}^{d}} c_{J} z^{J}: c_{J} \in \mathbb{C}\right\} .
$$

Recent results in multivariate approximation theory ([7, [9]) suggest that it is useful to generalize the notion of degree. Let $C \subset \mathbb{R}_{+}^{N}$ be a compact convex set which we will assume contains $\Sigma$. Given $n \in \mathbb{N}$, define $\operatorname{Poly}(n C)$ to be the collection of polynomials defined as in 1.2 above with $n C$ replacing $n \Sigma$ in the sum over the exponents $J$. The generalized degree (depending on $C$, or $C$-degree) of a nonconstant polynomial $p$ is given by

$$
\operatorname{deg}_{C}(p):=\min \{n \in \mathbb{N}: p \in \operatorname{Poly}(n C)\},
$$

which clearly reduces to $\operatorname{deg}(p)$ when $C=\Sigma$.

The classical theory of polynomial approximation and interpolation in $\mathbb{C}^{N}$ involves fundamental notions of pluripotential theory: the Siciak-Zaharjuta extremal function associated to a compact set $K \subset \mathbb{C}^{N}$

$$
V_{K}(z):=\sup \left\{\frac{1}{\operatorname{deg} p} \log ^{+}|p(z)|:\|p\|_{K} \leq 1\right\},
$$

complex equilibrium measure $\left(d d^{c} V_{K}\right)^{N}$, and Fekete-Leja transfinite diameter. All of these quantities are fundamentally related to the classical notion of degree. They must be appropriately generalized if one wants to prove approximation theorems involving $\operatorname{deg}_{C}$ or $\operatorname{Poly}(n C)$.

This motivates recent work on the so-called $C$-pluripotential theory. The spaces $\operatorname{Poly}(n C)$ and $C$-extremal function

$$
V_{C, K}(z):=\sup \left\{\frac{1}{\operatorname{deg}_{C} p} \log ^{+}|p(z)|:\|p\|_{K} \leq 1\right\}
$$

Key words and phrases. Chebyshev constant, Chebyshev transform, convex body, polynomial degree, submultiplicative function, transfinite diameter. 
were defined in [1] and further studied in [7] ${ }^{*}$ The equilibrium measure $\left(d d^{c} V_{C, K}\right)^{N}$ and $C$-transfinite diameter $\delta_{C}(K)$ were studied in [2], using the $L^{2}$ energy methods of Berman-Boucksom pioneered in [3], [4].

The Berman-Boucksom methods work more naturally in a general weighted setting involving an admissible weight function $w: K \rightarrow[0, \infty)$ and its logarithm $Q=-\log |w|$. These give weighted versions of all of the above notions, denoted $V_{C, K, Q}(z),\left(d d^{c} V_{C, K, Q}\right)^{N}$, and $\delta_{C}^{w}(K)$. Setting $w \equiv 1$ recovers the unweighted case.

In this paper, we study the weighted $C$-transfinite diameter $\delta_{C}^{w}(K)$. Our main theorem is the following.

Theorem (Theorem 4.6). Let $K \subset \mathbb{C}^{N}$ be compact and $w: K \rightarrow[0, \infty)$ an admissible weight. Then we have the formula

$$
\delta_{C}^{w}(K)^{A_{N}}=\exp \left(\frac{1}{\operatorname{vol}_{N}(C)} \int_{\operatorname{int}(C)} \log T_{\prec}^{w}(K, \theta) d \theta\right) .
$$

Here, $\operatorname{vol}_{N}$ denotes real $N$-dimensional volume, $d \theta$ the corresponding volume measure where $\theta=\left(\theta_{1}, \ldots, \theta_{N}\right)$ are coordinates in $\mathbb{R}^{N}$, and $A_{N}$ is a normalization constant. The quantity $T_{\prec}^{w}(K, \theta)$ is called a directional Chebyshev constant (for the direction $\theta$ ), and the function

$$
\operatorname{int}(C) \ni \theta \mapsto \log T_{\prec}^{w}(K, \theta) \in[0, \infty)
$$

is called the Chebyshev transform. (We follow the terminology in [6] and [10].)

In the next section (Section 2), we construct directional Chebyshev constants and the Chebyshev transform by a limiting process using submultiplicative functions (see Definition 2.7). Our overall method is standard, but we encounter a new technicality in the $C$-theory. The grevlex ordering on monomials, denoted in this paper by $\prec$ (see Definition 2.1), satisfies:

(1) If $\operatorname{deg}\left(z^{\alpha}\right)<\operatorname{deg}\left(z^{\beta}\right)$ then $z^{\alpha} \prec z^{\beta}$.

(2) If $z^{\alpha} \prec z^{\beta}$ then $z^{\alpha+\gamma} \prec z^{\beta+\gamma}$ for each $\gamma$.

Property (1) is needed for theorems that involve the notion of degree, and property (2) is needed to create submultiplicative functions. If we replace deg with $\operatorname{deg}_{C}$, then statement (1) fails for the grevlex ordering $\prec$; consider replacing it with some global ordering on monomials (denoted by, say, $\prec_{C}$ ) such that the following analog holds:

$\left(1_{C}\right)$ If $\operatorname{deg}_{C}\left(z^{\alpha}\right) \prec_{C} \operatorname{deg}_{C}\left(z^{\beta}\right)$ then $z^{\alpha} \prec_{C} z^{\beta}$.

It is easy to verify that in cases where $C$ is not a simplex, one can find $\alpha, \beta, \gamma$ such that $z^{\alpha} \prec_{C} z^{\beta}$ but $z^{\alpha+\gamma} \nprec_{C} z^{\beta+\gamma}$, i.e., the corresponding statement $\left(2_{C}\right)$ fails. However, to define directional Chebyshev constants via a limiting process one can get away with a weaker property $\left.\right|^{\dagger}$

In Section 2 we use the grevlex ordering $\prec$ to construct a submultiplicative function using discrete Chebyshev constants $T_{k}^{\prec}\left(\nu_{k}, \alpha\right)$. We construct directional Chebyshev constants $T_{\prec}^{w}(K, \theta)$ by a limiting process as $k \rightarrow \infty$. Then in Section 3 , we construct discrete Chebyshev constants $T_{k}^{C}\left(\nu_{k}, \alpha\right)$ using a global ordering $\prec_{C}$ satisfying $\left(1_{C}\right)$, as well as the associated directional Chebyshev constants $T_{C}^{w}(K, \theta)$. The ordering $\prec_{C}$ needs to satisfy a certain technical property (property $(\dagger)$, that holds for a generic convex body $C$ ) in order to prove that $T_{C}^{w}(K, \theta)$ is given by a well-defined limit

\footnotetext{
*In those papers the convex body is denoted $P$ and the terminology $P$-pluripotential theory, $P$-extremal function, etc. is used.

${ }^{\dagger}$ But note that submultiplicativity is needed to deduce other properties of these constants, cf. Lemmas 2.9 and 2.10

${ }_{\text {} W e}$ conjecture that the limit exists in general.
} 
In Section 4, Theorem 4.6 is proved by suitably adapting the arguments in 8 . Then, the same estimates and some measure-theoretic arguments yield a similar theorem with $\log T_{C}^{w}(K, \theta)$ replacing $\log T_{\prec}^{w}(K, \theta)$ in the integral formula.

Finally, in Section 5, we generalize the construction of Leja points, using the ordering $\prec_{C}$. In the unweighted case $(w \equiv 1)$ we can use our estimates in Section 4 to deduce the asymptotic behaviour of these so-called $C$-Leja points. We show that in the limit, they give the $C$-transfinite diameter.

\section{Chebyshev constants from a submultiplicative function}

In this section we construct a submultiplicative function from sup norms of monic polynomials. We then construct directional Chebyshev constants using this function. The basic properties of this construction are well-known, going back to Zaharjuta 11. They are given in Lemma 2.9, whose proof is omitted $\$$

We assume throughout that our convex body $C \subset \mathbb{R}_{+}^{N}$ contains the simplex $\Sigma$ defined as in 1.1. By a calculation,

$$
r_{1} C+r_{2} C \subseteq\left(r_{1}+r_{2}\right) C
$$

holds for $r_{1}, r_{2} \in \mathbb{R}_{+}$.

When $k \in \mathbb{Z}_{+}$we obtain a grading of the polynomials $\mathbb{C}[z]=\bigcup_{k} \operatorname{Poly}(k C)$, and

$$
\operatorname{Poly}\left(k_{1} C\right) \operatorname{Poly}\left(k_{2} C\right) \subseteq \operatorname{Poly}\left(\left(k_{1}+k_{2}\right) C\right), \quad k_{1}, k_{2} \in \mathbb{Z}_{+}
$$

follows from (2.1).

Definition 2.1. Let $\prec$ denote the grevlex ordering on $\mathbb{Z}^{N}$, in which we set $\alpha \prec \beta$ if

- $|\alpha|<|\beta|$; or

- $|\alpha|=|\beta|$, and there exists $k \in\{1, \ldots, N\}$ such that $\alpha_{k}<\beta_{k}$ and $\alpha_{j}=\beta_{j}$ for all $j<k$.

For monomials, we set $z^{\alpha} \prec z^{\beta}$ if $\alpha \prec \beta$.

It is straightforward to verify that $\prec$ is compatible with addition.

Lemma 2.2. If $\alpha \prec \beta$ then $\alpha+\gamma \prec \beta+\gamma \prec \beta+\delta$ for all $\gamma, \delta \in \mathbb{N}^{N}$ with $\gamma \prec \delta$.

We next construct classes of monic polynomials. Given $k \in \mathbb{N}$ and $\alpha \in\left(k C \cap \mathbb{Z}^{N}\right)$, let

$$
\mathcal{M}_{k}^{\prec}(\alpha):=\left\{p \in \operatorname{Poly}(k C): p(z)=z^{\alpha}+\sum_{\substack{\beta \in k C \cap \mathbb{Z}^{N} \\ \beta \prec \alpha}} a_{\beta} z^{\beta}\right\} .
$$

Lemma 2.3. Let $C \subset \mathbb{R}_{+}^{N}$ be a convex body containing the standard simplex $\Sigma$, and let $k_{1}, k_{2} \in \mathbb{N}$. Then

$$
\mathcal{M}_{k_{1}}^{\prec}\left(\alpha_{1}\right) \mathcal{M}_{k_{2}}^{\prec}\left(\alpha_{2}\right) \subseteq \mathcal{M}_{k_{1}+k_{2}}^{\prec}\left(\alpha_{1}+\alpha_{2}\right)
$$

for all $\alpha_{1} \in k_{1} C$ and $\alpha_{2} \in k_{2} C$.

$$
\begin{gathered}
\text { Proof. Let } p=z^{\alpha_{1}}+\sum_{\substack{\beta \in k_{1} C \\
\beta \prec \alpha_{1}}} a_{\beta} z^{\beta} \text { and } q=z^{\alpha_{2}}+\sum_{\substack{\gamma \in k_{2} C \\
\gamma \prec \alpha_{2}}} c_{\gamma} z^{\gamma} \text {. Write } \\
p q=z^{\alpha_{1}+\alpha_{2}}+\sum_{\beta, \gamma} a_{\beta} c_{\gamma} z^{\beta+\gamma} .
\end{gathered}
$$

By 2.1), $\beta+\gamma \in\left(k_{1}+k_{2}\right) C \cap \mathbb{Z}^{N}$ for all $\beta, \gamma$; and by Lemma 2.2. $\alpha_{1}+\alpha_{2} \prec \beta+\gamma$ for all $\beta, \gamma$ in the sum. Hence $p q \in \mathcal{M}_{k_{1}+k_{2}}^{\prec}\left(\alpha_{1}+\alpha_{2}\right)$.

\footnotetext{
§But see the paragraph above Lemmas 1.1-1.2 in [6].
} 
Recall that $p \in \operatorname{Poly}(k C)$ may be identified with the (entire) function $a \mapsto$ $p(a)$ given by the usual evaluation of polynomials. But we can also fix a function $\varphi: \mathbb{C}^{N} \rightarrow \mathbb{C}$ (a weight) and identify $p \in \operatorname{Poly}(k C)$ with $a \mapsto \varphi(a)^{k} p(a)$ (a weighted evaluation), and use this to define function-theoretic norms.

In what follows, $\nu_{k}$ denotes a norm on Poly $(k C)$. Examples of norms are:

(1) $\nu_{k}(p)=\left\|\varphi^{k} p\right\|_{K}$, where $K \subset \mathbb{C}^{n}$ is compact and $\varphi: K \rightarrow \mathbb{C}$ is continuous.

(2) $\nu_{k}(p)=\left(\int_{K}\left|\varphi^{k} p\right|^{2} d \mu\right)^{1 / 2}$, where $\varphi, K$ are as in (1) and $\mu$ is a finite measure on $K$.

Suppose for each $k \in \mathbb{N}, \nu_{k}$ is as in either (1) or (2). Then we have the following.

Lemma 2.4. Let $k, m \in \mathbb{N}$, let $p \in \operatorname{Poly}(k C)$ and let $q \in \operatorname{Poly}(m C)$. Then $p q \in \operatorname{Poly}((k+m) C)$ and

$$
\nu_{k+m}(p q) \leq \nu_{k}(p) \nu_{m}(q) .
$$

Proof. If $\nu_{k}, \nu_{m}$ are as in (1), then $\left\|\varphi^{k+m} p q\right\|_{K} \leq\left\|\varphi^{k} p\right\|_{K}\left\|\varphi^{m} q\right\|_{K}$ and the conclusion follows. If the norms are as in (2), use the Cauchy-Schwarz inequality.

For each $k \in \mathbb{N}$ let $\nu_{k}$ be a norm on $\operatorname{Poly}(k C)$. The sequence of norms $\left\{\nu_{k}\right\}_{k=1}^{\infty}$ is submultiplicative if 2.4 holds for every $k, m \in \mathbb{N}$.

Definition 2.5. Define the discrete Chebyshev constant $T_{k}^{\prec}\left(\nu_{k}, \alpha\right)$ by

$$
T_{k}^{\prec}\left(\nu_{k}, \alpha\right):=\inf \left\{\nu_{k}(p): p \in \mathcal{M}_{k}^{\prec}(\alpha)\right\}^{1 / k} .
$$

A polynomial $t$ for which $\operatorname{deg}_{C}(t)=k$ and $T_{k}^{\prec}\left(\nu_{k}, \alpha\right)=\nu_{k}(t)^{1 / k}$ will be called a Chebyshev polynomial of degree $k$.

Lemma 2.6. If $\left\{\nu_{k}\right\}_{k=1}^{\infty}$ is a submultiplicative sequence of norms, then

$$
T_{k_{1}+k_{2}}^{\prec}\left(\nu_{k_{1}+k_{2}}, \alpha_{1}+\alpha_{2}\right)^{k_{1}+k_{2}} \leq T_{k_{1}}^{\prec}\left(\nu_{k_{1}}, \alpha_{1}\right)^{k_{1}} T_{k_{2}}^{\prec}\left(\nu_{k_{2}}, \alpha_{2}\right)^{k_{2}} .
$$

Proof. Let $t_{k_{1}}, t_{k_{2}}$ be polynomials satisfying

$$
\nu_{k_{1}}\left(t_{k_{1}}\right)=T_{k_{1}}^{\prec}\left(\nu_{k_{1}}, \alpha_{1}\right)^{k_{1}} \text { and } \nu_{k_{2}}\left(t_{k_{2}}\right)=T_{k_{2}}^{\prec}\left(\nu_{k_{2}}, \alpha_{2}\right)^{k_{2}} \text {. }
$$

Then $t_{k_{1}} t_{k_{2}} \in \mathcal{M}^{\prec}\left(k_{1}+k_{2}, \alpha_{1}+\alpha_{2}\right)$ by Lemma 2.2, and

$$
T_{k_{1}+k_{2}}^{\prec}\left(\nu_{k_{1}+k_{2}}, \alpha_{1}+\alpha_{2}\right)^{k_{1}+k_{2}} \leq \nu_{k_{1}+k_{2}}\left(t_{k_{1}} t_{k_{2}}\right) \leq \nu_{k_{1}}\left(t_{1}\right) \nu_{k_{2}}\left(t_{k_{2}}\right) .
$$

by submultiplicativity. Now put 2.5) into the above.

Let $\Omega \subset \mathbb{Z}_{+} \times \mathbb{Z}_{+}^{N}$ be a set that is closed under addition (i.e., $(k, \alpha),(l, \beta) \in \Omega$ implies $(k+l, \alpha+\beta) \in \Omega)$.

Definition 2.7. A function $Y: \Omega \rightarrow[0, \infty)$ is called submultiplicative if

$$
Y(k+l, \alpha+\beta) \leq Y(k, \alpha) Y(l, \beta) .
$$

As an immediative consequence of Lemma 2.6, we have the following.

Corollary 2.8. The function $(k, \alpha) \mapsto T_{k}\left(\nu_{k}, \alpha\right)^{k}$ is submultiplicative on the set

$$
\Omega_{C}:=\left\{(k, \alpha): k \in \mathbb{Z}_{+}, \alpha \in k C \cap \mathbb{Z}^{N}\right\} .
$$

A submultiplicative function on $\Omega_{C}$ yields a real convex function on $\operatorname{int}(C)$ by a limiting process.

Lemma 2.9. Let $Y: \Omega_{C} \rightarrow[0, \infty)$ be submultiplicative. 
(1) The limit

$$
Y_{\infty}(\theta):=\lim _{\substack{k \rightarrow \infty \\ \alpha / k \rightarrow \theta}} Y(k, \alpha)^{1 / k}
$$

exists for all $\theta \in \operatorname{int}(C)$.

(2) We have

$$
\begin{aligned}
& Y_{\infty}(t \theta+(1-t) \phi) \leq Y_{\infty}(\theta)^{t} Y_{\infty}(\phi)^{1-t} \text { for all } \theta, \phi \in \operatorname{int}(C) \text { and } t \in[0,1] . \\
& \text { Hence } \operatorname{int}(C) \ni \theta \mapsto \log Y_{\infty}(\theta) \in \mathbb{R} \text { is a convex function. }
\end{aligned}
$$

In particular, part (2) shows that $\theta \mapsto \log Y_{\infty}(\theta)$ is continuous, and hence uniformly continuous on compact subsets of $\mathbb{R}_{+}^{N}$. An exercise in the triangle inequality then yields the following.

Lemma 2.10. Let $Q \subset \operatorname{int}(C)$ be compact. For each $k \in \mathbb{N}$, consider the nodes $Q \cap \frac{1}{k} \mathbb{Z}^{N}$ under some enumeration $\left\{\theta_{j}\right\}_{j=1}^{N_{k}}$, with $\frac{1}{k} \alpha_{j, k}=\theta_{j}$. Then

$$
\sup \left\{\left|\log Y\left(\alpha_{j, k}\right)^{1 / k}-\log Y_{\infty}\left(\theta_{j}\right)\right|: j \in\left\{1, \ldots, N_{k}\right\}\right\} \longrightarrow 0 \text { as } k \rightarrow \infty .
$$

Fix a compact set $K \subset \mathbb{C}^{N}$ and a continuous weight $w: K \rightarrow[0, \infty)$. With the submultiplicative sequence of norms $\nu_{k}(p):=\left\|w^{k} p\right\|_{K}$, construct the submultiplicative function $Y(k, \alpha):=T_{k}^{\prec}\left(\nu_{k}, \alpha\right)^{k}$ on $\Omega_{C}$. Then for each $\theta \in \operatorname{int}(C)$, set $T_{\prec}^{w}(K, \theta):=Y_{\infty}(\theta)$, as constructed in Lemma 2.9. This yields the following corollary.

Corollary 2.11. Lemmas 2.9 and 2.10 hold with $T_{k}^{\prec}\left(\nu_{k}, \alpha\right)^{k}, T_{\prec}^{w}(K, \theta)$ replacing $Y(k, \alpha), Y_{\infty}(\theta)$.

Definition 2.12. The number $T_{\prec}^{w}(K, \theta)$ is called a directional Chebyshev constant (with direction $\theta)$ associated to $(K, w)$, and the function $\theta \mapsto \log T_{\prec}^{w}(K, \theta)$ is called the Chebyshev transform.

\section{Chebyshev constants COMpatible With Generalized Degree}

Now consider a modified grevlex ordering that is compatible with the grading given by the convex body $C$. First, for $\alpha \in \mathbb{Z}_{+}^{N}$ define

$$
r(\alpha):=\inf \left\{r \in \mathbb{R}_{+}: \alpha \in r C\right\} .
$$

Then for $j \in \mathbb{Z}_{+}, r(j \alpha)=j r(\alpha)$ is immediate, and

$$
r(\alpha+\beta) \leq r(\alpha)+r(\beta), \quad \alpha, \beta \in \mathbb{Z}_{+}^{N}
$$

follows from 2.1). Thus

$$
r(j \alpha+k \beta) \leq j r(\alpha)+k r(\beta), \quad \alpha, \beta \in \mathbb{Z}_{+}^{N} \text { and } j, k \in \mathbb{Z}_{+} .
$$

Definition 3.1. Define $\prec_{C}$ on $\mathbb{Z}_{+}^{N}$ by setting $\alpha \prec_{C} \beta$ (and equivalently, $z^{\alpha} \prec_{C} z^{\beta}$ ) if

- $r(\alpha)<r(\beta)$; or

- $r(\alpha)=r(\beta)$ and $z^{\alpha} \prec z^{\beta}$ (where as before, $\prec$ denotes grevlex).

This is compatible with the grading: if $\alpha \prec_{C} \beta$ then $r(\alpha) \leq r(\beta)$, so

$$
\operatorname{deg}_{C}\left(z^{\alpha}\right)=\lceil r(\alpha)\rceil \leq\lceil r(\beta)\rceil=\operatorname{deg}_{C}\left(z^{\beta}\right) .
$$

For $\alpha \in k C$, define the class of monic polynomials

$$
\mathcal{M}_{k}^{C}(\alpha):=\left\{p \in \operatorname{Poly}(k C): p(z)=z^{\alpha}+\sum_{\substack{\beta \in k C \cap \mathbb{Z}^{N} \\ \beta \prec C}} a_{\beta} z^{\beta}\right\}
$$


and the associated (discrete) Chebyshev constant

$$
T_{k}^{C}\left(\nu_{k}, \alpha\right):=\inf \left\{\nu_{k}(p): p \in \mathcal{M}_{k}^{C}(\alpha)\right\}^{1 / k},
$$

where $\nu_{k}(p)=\left\|w^{k} p\right\|_{K}$, as before.

As mentioned in the Introduction: $T_{k}^{C}\left(\nu_{k}, \alpha\right)$ does not yield a submultiplicative function (unlike $T_{k}^{\prec}\left(\nu_{k}, \alpha\right)$ ), essentially because $\mathcal{M}_{k}^{C}(\alpha)$ uses the ordering $\prec_{C}$ which is not compatible with addition of exponents. Hence we cannot apply Lemmas 2.9 and 2.10 directly. However, we do have the following.

Lemma 3.2. Let $\alpha \in k C$. Then $p \in \mathcal{M}_{k}^{C}(\alpha)$ implies $p^{j} \in \mathcal{M}_{j k}^{C}(j \alpha)$ for $j \in \mathbb{Z}_{+}$.

Proof. Write

$$
p(z)=z^{\alpha}+\sum_{\substack{\beta \in k C \cap \mathbb{Z}^{N} \\ \beta \prec C}} a_{\beta} z^{\beta} .
$$

We claim that the leading term of $p^{j}$ with respect to $\prec_{C}$ is $z^{j \alpha}$. The monomial of any other term is of the form $z^{k \alpha+(j-k) \beta}$ for some $\beta \prec_{C} \alpha$ and $k<j$. If $r(\beta)<r(\alpha)$ then

So $k \alpha+(j-k) \beta \prec_{C} j \alpha$.

$$
r(k \alpha+(j-k) \beta) \leq k r(\alpha)+(j-k) r(\beta)<j r(\alpha)=r(j \alpha),
$$

If $r(\beta)=r(\alpha)$ then similarly, $r(k \alpha+(j-k) \beta) \leq r(j \alpha)$. We also have $\beta \prec \alpha$. The latter gives $k \alpha+(j-k) \beta \prec k \alpha$ since grevlex is compatible with addition. Altogether, we can conclude that $k \alpha+(j-k) \beta \prec_{C} j \alpha$ holds in all cases. Thus the leading term of $p^{j}$ is $z^{j \alpha}$, so $p^{j} \in \mathcal{M}_{j k}^{C}(j \alpha)$.

We need a slightly stronger property than $p \in \mathcal{M}_{k}^{C}(\alpha) \Longrightarrow p^{j} \in \mathcal{M}_{j k}^{C}(j \alpha)$ to apply the standard argument showing that the constants $T_{k}^{C}\left(\nu_{k}, \alpha\right)$ give directional limits:

(†) Given $k, l \in \mathbb{Z}_{+}, \alpha \in k C$, and $p \in \mathcal{M}_{k}^{C}(\alpha)$, there exists $j_{0} \in \mathbb{Z}_{+}$such that for any $\delta \in l C$,

$$
z^{\delta} p^{j} \in \mathcal{M}_{j k+l}^{C}(j \alpha+\delta) \text { for all } j \geq j_{0} .
$$

If $C$ is a simplex, then $\prec_{C}$ is actually submultiplicative, which easily implies $(\dagger)$. We can see this by elementary geometry. Put $r_{1}=r(\alpha)$ and $r_{2}=r(\alpha+\delta)$. Then $\partial\left(r_{1} C\right) \cap \mathbb{R}_{+}^{N}$ and $\partial\left(r_{2} C\right) \cap \mathbb{R}_{+}^{N}$ are on parallel hyperplanes, so translation by $\delta$ sends all points of the first hyperplane into the second. It follows that $r(\beta) \leq r(\alpha)$ implies $r(\beta+\delta) \leq r(\alpha+\delta)$, and hence, $\beta \prec_{C} \alpha$ implies $\beta+\delta \prec_{C} \alpha+\delta$.

Another case for which $(\dagger)$ holds is when the scaled copies $r C\left(r \in \mathbb{R}_{+}\right)$cover new points of $\mathbb{Z}_{+}^{N}$ one at a time as $r \rightarrow \infty$. In other words, whenever $\alpha \neq \beta$ we have $r(\alpha) \neq r(\beta)$. (Clearly, this condition holds for a generic convex body $C$.)

To verify $(\dagger)$ in this case, let $N(p)$ be the Newton polytope of $p \in \mathcal{M}_{k}^{C}(\alpha)$, i.e., the convex hull in $\mathbb{R}_{+}^{N}$ of all $\beta \in \mathbb{Z}_{>0}^{N}$ such that $p$ contains the monomial $z^{\beta}$. Put $r=r(\alpha)$; then by hypothesis, $r(\beta)<r$ for all other terms of $p$ (since $z^{\alpha}$ is the leading term), so $\alpha$ is the only point of $N(p)$ on $\partial(r C) \cap \mathbb{R}_{+}^{N}$.

For $s>0$, a translation of $N(p)$ by $s \delta$ takes $\alpha$ to a point $\alpha+s \delta$ on $\partial(\tilde{r} C)$ for some $\tilde{r}>r$. If we take $s$ sufficiently close to zero, then only points in a small neighborhood of $\alpha$ will move into the complement of $r C$. We can consider a neighborhood small enough so that $\partial(r C)$ and $\partial(\tilde{r} C)$ are approximated by parallel hyperplanes. Then, a translation of $N(p)$ by $s \delta$ sends $\alpha$ to $\partial(\tilde{r} C)$, but all other points of $N(p)$ remain in the interior of $\tilde{r} C$.

Now consider taking $s=\frac{1}{j}$ for $j \in \mathbb{Z}_{+}$sufficiently large. Next, note that $N\left(p^{j}\right)=$ $j N(p)$ by a straightforward calculation. With the polynomial $p^{j}$, everything in the previous paragraph scales by a factor of $j$, i.e., $N\left(p^{j}\right)=j N(p) \subset j r C$, and $j \alpha+\delta$ 
is the only point of $N\left(p^{j}\right)$ that translates to $\partial(j \tilde{r} C)$; all other points remain in the interior of $j \tilde{r} C$. See the picture below.

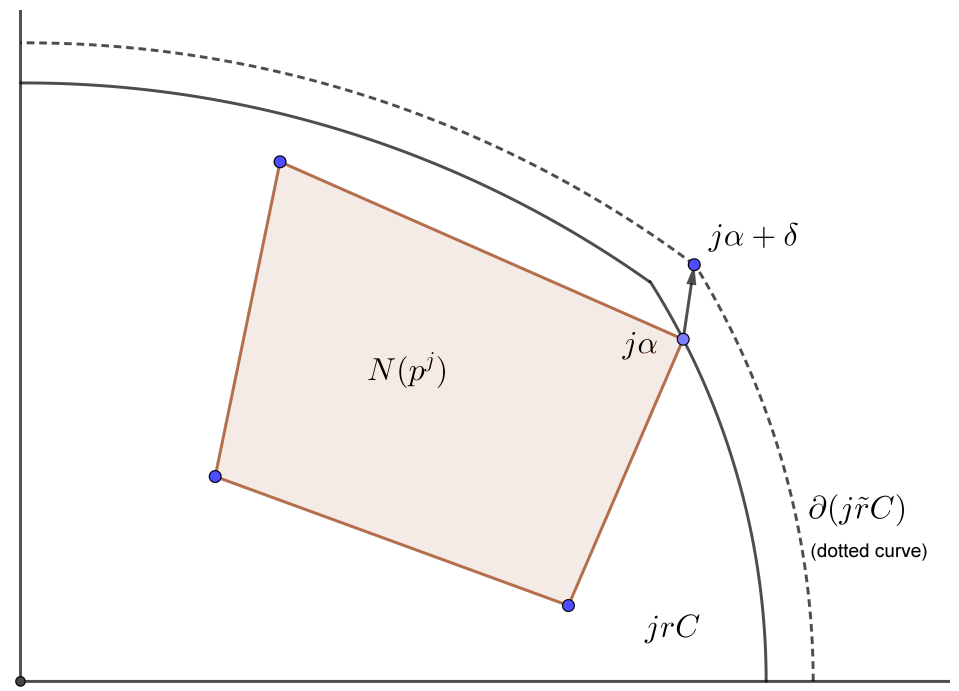

We have $N\left(p^{j}\right)+\delta=N\left(z^{\delta} p^{j}\right)$, so the leading term of $z^{\delta} p^{j}$ is given by $z^{\delta+j \alpha}$. Thus if $z^{\alpha}$ is the leading monomial of $p$, then $z^{j \alpha+\delta}$ is the leading monomial of $z^{\delta} p^{j}$, i.e., $z^{\delta} p^{j} \in \mathcal{M}_{j k+l}^{C}(j \alpha+\delta)$. This verifies condition ( $\left.\dagger\right)$.

Applying the argument of Lemma 1 in [11, we have the following.

Lemma 3.3. Suppose condition ( $\dagger$ ) holds for $C$. Then the limit

$$
T_{C}^{w}(K, \theta):=\lim _{\substack{k \rightarrow \infty \\ \alpha / k \rightarrow \theta}} T_{k}^{C}\left(\nu_{k}, \alpha\right)
$$

exists for all $\theta \in \operatorname{int}(C)$.

We leave it as an open problem to relate $T_{\prec}^{w}(K, \theta)$ to $T_{C}^{w}(K, \theta)$. Are these directional Chebyshev constants equal?

\section{VANDERMONDE DETERMINANT ESTIMATES}

In this section, the compact set $K$ and continuous weight $w: K \rightarrow[0, \infty)$ are fixed. We assume condition $(\dagger)$ of the previous section holds for $C$. We also use the following notation:

$$
\begin{aligned}
M_{k} & =\text { number of points in } k C \cap \mathbb{Z}^{N}=\operatorname{dim} \operatorname{Poly}(k C), \\
h_{k} & =\text { number of points in }(k C \backslash(k-1) C) \cap \mathbb{Z}^{N}, \\
L_{k} & =\sum_{\alpha \in k C} \operatorname{deg}\left(z^{\alpha}\right) .
\end{aligned}
$$

Remark 4.1. Note that $L_{k}$ is the sum of the ordinary degrees of the monomials, not the $C$-degrees. The normalization in equation 4.3 below uses an $L_{k}$-th root so that transfinite diameter scales like a length.

We have the following limits. The first two are easy, and the last one is a bit more involved - an explicit calculation is given in 2] (see Remark 2.17 of that paper.

Lemma 4.2. As $k \rightarrow \infty$,

$$
\frac{h_{k}}{M_{k}} \rightarrow 0, \quad\left(M_{k} !\right)^{1 / k M_{k}} \rightarrow 1, \quad \text { and } \frac{L_{k}}{k M_{k}} \rightarrow A_{N},
$$


where

$$
A_{N}=\frac{1}{\operatorname{vol}_{N}(C)} \int_{C}\left(\theta_{1}+\cdots+\theta_{N}\right) d \theta .
$$

Here $d \theta=d \theta_{1} \wedge \cdots \wedge d \theta_{N}$ denotes the $N$-dimensional volume measure in $\mathbb{R}^{N}$ with coordinates $\theta=\left(\theta_{1}, \ldots, \theta_{N}\right)$.

Definition 4.3. Let $k \in \mathbb{N}$, and let $\{\alpha(j)\}_{j=1}^{M_{k}}$ be an enumeration of $k C \cap \mathbb{Z}^{N}$, so that $\left\{z^{\alpha(j)}\right\}_{j=1}^{M_{k}}$ is a basis of $\operatorname{Poly}(k C)$. Given a finite collection of points $\left\{\zeta_{1}, \ldots, \zeta_{s}\right\}$ with $s \leq M_{k}$, define the Vandermonde matrix

$$
V M_{k}^{w}\left(\zeta_{1}, \ldots, \zeta_{s}\right):=\left[\begin{array}{ccc}
w\left(\zeta_{1}\right)^{k} z^{\alpha_{1}}\left(\zeta_{1}\right) & \cdots & w\left(\zeta_{s}\right)^{k} z^{\alpha_{1}}\left(\zeta_{s}\right) \\
\vdots & \ddots & \vdots \\
w\left(\zeta_{1}\right)^{k} z^{\alpha_{s}}\left(\zeta_{1}\right) & \cdots & w\left(\zeta_{s}\right)^{k} z^{\alpha_{s}}\left(\zeta_{s}\right)
\end{array}\right]=\left[w\left(\zeta_{l}\right)^{k} z^{\alpha_{j}}\left(\zeta_{l}\right)\right]_{j, l=1}^{s},
$$

as well as the Vandermonde determinant

$$
V D M_{k}^{w}\left(\zeta_{1}, \ldots, \zeta_{s}\right):=\operatorname{det}\left(V M_{k}^{w}\left(\zeta_{1}, \ldots, \zeta_{s}\right)\right) .
$$

For a set $K \subset V$, define

$$
V_{k}^{w}(K, s):=\sup \left\{\left|V D M_{k}^{w}\left(\zeta_{1}, \ldots, \zeta_{s}\right)\right|:\left\{\zeta_{1}, \ldots, \zeta_{s}\right\} \subset K\right\} .
$$

The quantity

$$
\delta_{C, k}^{w}(K):=\left(V_{k}^{w}\left(K, M_{k}\right)\right)^{1 / L_{k}} \text { is the } k \text {-th order } C \text {-diameter of } K,
$$

and

$$
\delta_{C}^{w}(K):=\limsup _{k \rightarrow \infty} \delta_{C, k}^{w}(K) \text { is the (weighted) } C \text {-transfinite diameter of } K \text {. }
$$

We compute upper and lower bounds for $V_{k}^{w}\left(K, M_{k}\right)$.

Lemma 4.4. Fix $k \in \mathbb{N}$. The inequality

$$
V_{k}^{w}\left(K, M_{k}\right) \leq M_{k} ! \prod_{j=1}^{M_{k}} T_{k}^{\prec}\left(\nu_{k}, \alpha(j)\right)^{k}
$$

holds. The same inequality holds if we replace each factor $T_{k}^{\prec}\left(\nu_{k}, \alpha(j)\right)$ on the right-hand side by $T_{k}^{C}\left(\nu_{k}, \alpha(j)\right)$.

Proof. Enumerate the monomials $\left\{z^{\alpha(j)}\right\}_{j=1}^{M_{k}}$ according to the grevlex ordering, and for each $j \in\left\{1, \ldots, M_{k}\right\}$, let

$$
\mathbf{t}_{j}(z)=z^{\alpha(j)}+\sum_{l \prec j} c_{l} z^{\alpha(l)}
$$

denote the Chebyshev polynomial in $\mathcal{M}_{k}(\alpha(j))$, i.e., $\left\|w^{k} \mathbf{t}_{j}\right\|_{K}=T_{k}^{\prec}(K, \alpha(j))^{k}$. Now choose a set of $M_{k}$ points such that $V_{k}^{w}\left(K, M_{k}\right)=\left|V D M_{k}^{w}\left(\zeta_{1}, \ldots, \zeta_{M_{k}}\right)\right|$; then

$$
V D M_{k}^{w}\left(\zeta_{1}, \ldots, \zeta_{M_{k}}\right)=\operatorname{det}\left[\begin{array}{ccc}
w^{k}\left(\zeta_{1}\right) z^{\alpha(1)}\left(\zeta_{1}\right) & \cdots & w^{k}\left(\zeta_{M_{k}}\right) z^{\alpha(1)}\left(\zeta_{M_{k}}\right) \\
\vdots & \ddots & \vdots \\
w^{k}\left(\zeta_{1}\right) z^{\alpha\left(M_{k}-1\right)}\left(\zeta_{1}\right) & \cdots & w^{k}\left(\zeta_{M_{k}}\right) z^{\alpha\left(M_{k}-1\right)}\left(\zeta_{M_{k}}\right) \\
w^{k}\left(\zeta_{1}\right) \mathbf{t}_{M_{k}}\left(\zeta_{1}\right) & \cdots & w^{k}\left(\zeta_{1}\right) \mathbf{t}_{M_{k}}\left(\zeta_{M_{k}}\right)
\end{array}\right]
$$

since we may add multiples of other rows to the last, leaving the determinant unchanged. Expanding and using the triangle inequality, we obtain

$$
\begin{aligned}
V_{k}^{w}\left(K, M_{k}\right) & \leq \sum_{j=1}^{M_{k}}\left|w^{k}\left(\zeta_{j}\right) \mathbf{t}_{M_{k}}\left(\zeta_{j}\right)\right| \cdot\left|V D M_{k}^{w}\left(\zeta_{1}, \ldots, \zeta_{j-1}, \zeta_{j+1}, \ldots, \zeta_{M_{k}}\right)\right| \\
& \leq M_{k} \cdot T_{k}^{\prec}\left(\nu_{k}, \alpha\left(M_{k}\right)\right)^{k} V_{k}^{w}\left(K, M_{k}-1\right) .
\end{aligned}
$$


A similar argument may be used to obtain

$$
V_{k}^{w}\left(K, M_{k}-1\right) \leq\left(M_{k}-1\right) \cdot T_{k}^{\prec}\left(\nu_{k}, \alpha\left(M_{k}-1\right)\right)^{k} V_{k}^{w}\left(K, M_{k}-2\right),
$$

and further, $V_{k}^{w}(K, j) \leq j \cdot T_{k}^{\prec}\left(\nu_{k}, \alpha(j)\right)^{k} V_{k}^{w}(K, j-1)$ for any smaller $j$. Putting all of these inequalities together gives the desired inequality.

For the inequality involving the $T_{k}^{C}\left(\nu_{k}, \alpha(j)\right)^{k}$ constants, repeat the proof using $\prec_{C}$ to enumerate the monomials.

Lemma 4.5. Fix $k \in \mathbb{N}$. Then

$$
V_{k}^{w}\left(K, M_{k}\right) \geq \prod_{j=1}^{M_{k}} T_{k}^{\prec}\left(\nu_{k}, \alpha(j)\right)^{k} .
$$

The same result holds if we replace each factor $T_{k}^{\prec}\left(\nu_{k}, \alpha(j)\right)$ on the right-hand side by $T_{k}^{C}\left(\nu_{k}, \alpha(j)\right)$.

Proof. Fix $k \in \mathbb{N}$, and let $\left\{z^{\alpha(j)}\right\}_{j=1}^{M_{k}}$ be the enumeration of the monomials in Poly $(k C)$ according to grevlex, i.e., $z^{\alpha(j)} \prec z^{\alpha(k)}$ if $j<k$.

Let us introduce the following notation: for $j=1, \ldots, M_{k}$, set

$$
W_{j}\left(\zeta_{1}, \ldots, \zeta_{j}\right):=\left|\operatorname{det}\left[\begin{array}{ccc}
w^{k}\left(\zeta_{1}\right) z^{\alpha(1)}\left(\zeta_{1}\right) & \cdots & w^{k}\left(\zeta_{j}\right) z^{\alpha(1)}\left(\zeta_{j}\right) \\
\vdots & \ddots & \vdots \\
w^{k}\left(\zeta_{1}\right) z^{\alpha(j)}\left(\zeta_{1}\right) & \cdots & w^{k}\left(\zeta_{j}\right) z^{\alpha(j)}\left(\zeta_{j}\right)
\end{array}\right]\right|
$$

Observe that for any collection of points $\left\{\zeta_{1}, \ldots, \zeta_{M_{k}}\right\} \subseteq K$,

$$
V_{k}^{w}\left(K, M_{k}\right) \geq W_{M_{k}}\left(\zeta_{1}, \ldots, \zeta_{M_{k}}\right) .
$$

We now derive an inequality involving $W_{M_{k}-1}$. Fix $\left\{\zeta_{1}, \ldots, \zeta_{M_{k}-1}\right\} \subseteq K$. Then, by considering row operations as before, $W_{M_{k}}\left(\zeta_{1}, \ldots, \zeta_{M_{k}-1}, \eta\right)$ is equal to

$$
\operatorname{det}\left[\begin{array}{cccc}
w^{k}\left(\zeta_{1}\right) z^{\alpha(1)}\left(\zeta_{1}\right) & \cdots & w^{k}\left(\zeta_{M_{k}-1}\right) z^{\alpha(1)}\left(\zeta_{M_{k}-1}\right) & w^{k}(\eta) z^{\alpha(1)}(\eta) \\
\vdots & \ddots & \vdots & \vdots \\
w^{k}\left(\zeta_{1}\right) z^{\alpha\left(M_{k}-1\right)}\left(\zeta_{1}\right) & \cdots & w^{k}\left(\zeta_{M_{k}-1}\right) z^{\alpha\left(M_{k}-1\right)}\left(\zeta_{M_{k}-1}\right) & w^{k}(\eta) z^{\alpha\left(M_{k}-1\right)}(\eta) \\
w^{k}\left(\zeta_{1}\right) \mathbf{p}_{M_{k}}\left(\zeta_{1}\right) & \cdots & w^{k}\left(\zeta_{M_{k}-1}\right) \mathbf{p}_{M_{k}}\left(\zeta_{M_{k}-1}\right) & w^{k}(\eta) \mathbf{p}_{M_{k}}(\eta)
\end{array}\right] \mid
$$

for any $\mathbf{p}_{M_{k}} \in \mathcal{M}_{k}^{\prec}\left(\alpha\left(M_{k}\right)\right)$ and $\eta \in K$. We choose the particular multiples of previous rows that give a polynomial satisfying $\mathbf{p}_{M_{k}}\left(\zeta_{1}\right)=\cdots=\mathbf{p}_{M_{k}}\left(\zeta_{M_{k}-1}\right)=0$. Then $\mathbf{p}_{M_{k}} \in \mathcal{M}_{k}^{\prec}\left(\alpha\left(M_{k}\right)\right)$ and

$$
\begin{aligned}
& V_{k}^{w}\left(K, M_{k}\right) \\
& \quad \geq \operatorname{det}\left[\begin{array}{cccc}
w^{k}\left(\zeta_{1}\right) z^{\alpha(1)}\left(\zeta_{1}\right) & \ldots & w^{k}\left(\zeta_{M_{k}-1}\right) z^{\alpha(1)}\left(\zeta_{M_{k}-1}\right) & w^{k}(\eta) z^{\alpha(1)}(\eta) \\
\vdots & \ddots & \vdots & \vdots \\
w^{k}\left(\zeta_{1}\right) z^{\alpha\left(M_{k}\right)}\left(\zeta_{1}\right) & \ldots & w^{k}\left(\zeta_{M_{k}-1}\right) z^{\alpha\left(M_{k}\right)}\left(\zeta_{M_{k}-1}\right) & w^{k}(\eta) z^{\alpha\left(M_{k}\right)}(\eta) \\
0 & \ldots & 0 & w^{k}(\eta) \mathbf{p}_{M_{k}}(\eta)
\end{array}\right] \mid \\
& \quad=\left|w^{k}(\eta) \mathbf{p}_{M_{k}}(\eta)\right| \cdot\left|W_{1}\left(\zeta_{1}, \ldots, \zeta_{M_{k}-1}\right)\right| .
\end{aligned}
$$

Choose $\eta \in K$ that attains the sup norm, so that

$$
\left|w^{k}(\eta) \mathbf{p}_{M_{k}}(\eta)\right|=\left\|w^{k} \mathbf{p}_{M_{k}}\right\|_{K} \geq T_{k}^{\prec}\left(\nu_{k}, \alpha\left(M_{k}\right)\right)^{k} ;
$$

then

(4.4)

$V_{k}^{w}\left(K, M_{k}\right) \geq\left|W_{M_{k}}\left(\zeta_{1}, \ldots, \zeta_{M_{k}-1}, \eta\right)\right| \geq\left|W_{M_{k}-1}\left(\zeta_{1}, \ldots, \zeta_{M_{k}-1}\right)\right| T_{k}^{\prec}\left(\nu_{k}, \alpha\left(M_{k}\right)\right)^{k}$.

Observe that $\zeta_{1}, \ldots, \zeta_{M_{k}-1}$ are arbitrary points of $K$ in the above equation. Now consider fixing $\zeta_{1}, \ldots, \zeta_{M_{k}-2}$ and carry out a similar argument: construct a polynomial $\mathbf{p}_{M_{k}-1} \in \mathcal{M}_{k}^{\prec}\left(\alpha\left(M_{k}-1\right)\right)$ with $\mathbf{p}_{M_{k}-1}\left(\zeta_{1}\right)=\cdots=\mathbf{p}_{M_{k}-1}\left(\zeta_{M_{k}-2}\right)=0$, 
substitute it for $z^{\alpha\left(M_{k}-1\right)}$ in the second to last row, then choose the last point $\eta \in K$ such that $\left|w^{k}(\eta) \mathbf{p}_{M_{k}-1}(\eta)\right|=\left\|w^{k} \mathbf{p}_{M_{k}-1}\right\|_{K}$. This gives the inequality

$$
W_{M_{k}-1}\left(\zeta_{1}, \ldots, \zeta_{M_{k}-2}, \eta\right) \geq W_{M_{k}-2}\left(\zeta_{1}, \ldots, \zeta_{M_{k}-2}\right) T_{k}^{\prec}\left(\nu_{k}, \alpha\left(M_{k}-1\right)\right)^{k} .
$$

Use (4.4) to estimate the left-hand side of (4.5), observing that the upper bound on the left-hand side of (4.4) is valid for an arbitrary collection of $M_{k-1}$ points of $K$. Hence

$$
V_{k}^{w}\left(K, M_{k}\right) \geq W_{M_{k}-2}\left(\zeta_{1}, \ldots, \zeta_{M_{k}-2}\right) T_{k}^{\prec}\left(\nu_{k}, \alpha\left(M_{k}-1\right)\right)^{k} T_{k}^{\prec}\left(\nu_{k}, \alpha\left(M_{k}\right)\right)^{k} .
$$

Now it is easy to see that the argument can be iterated to obtain the estimate

$$
V_{k}^{w}\left(K, M_{k}\right) \geq W_{s}\left(\zeta_{1}, \ldots, \zeta_{s}\right)\left(\prod_{j=s+1}^{M_{k}} T_{k}^{\prec}\left(\nu_{k}, \alpha(j)\right)^{k}\right)
$$

for successively lower values of $s$. The first statement of the lemma is proved when $s=1$.

To get the inequality with each $T_{k}^{\prec}\left(\nu_{k}, \alpha(j)\right)$ replaced by $T_{k}^{C}\left(\nu_{k}, \alpha(j)\right)$, repeat the proof word for word, but enumerate the monomials using $\prec_{C}$.

Using these estimates, we can now prove transfinite diameter formulas. As mentioned in 4.1), the $L_{k}$-th root is chosen so that the quantities $\delta_{C, k}^{w}, \delta_{C}^{w}$ scale like a length. However it is more natural to take the $k M_{k}$-th root in the calculations that follow.

Theorem 4.6. The limit $D^{w}(K):=\lim _{k \rightarrow \infty}\left(V_{k}^{w}\left(K, M_{k}\right)\right)^{1 /\left(k M_{k}\right)}$, exists, and

$$
D^{w}(K)=\exp \left(\frac{1}{\operatorname{vol}_{N}(C)} \int_{\operatorname{int}(C)} \log T_{\prec}^{w}(K, \theta) d \theta\right) .
$$

The transfinite diameter is then given by

$$
\delta_{C}^{w}(K)=D^{w}(K)^{1 / A_{N}} .
$$

Proof. We have by Lemmas 4.4 and 4.5 .

$$
\prod_{j=1}^{M_{k}} T_{k}^{\prec}\left(\nu_{k}, \alpha(j)\right)^{k} \leq V_{k}^{w}\left(K, M_{k}\right) \leq M_{k} ! \prod_{j=1}^{M_{k}} T_{k}^{\prec}\left(\nu_{k}, \alpha(j)\right)^{k} .
$$

Let us take $k M_{k}$-th roots in this inequality and let $k \rightarrow \infty$. Since $\left(M_{k} !\right)^{1 /\left(k M_{k}\right)} \rightarrow 1$ as $k \rightarrow \infty$,

$$
D^{w}(K)=\lim _{k \rightarrow \infty}\left(V_{k}^{w}\left(K, M_{k}\right)\right)^{1 /\left(k M_{k}\right)}=\lim _{k \rightarrow \infty}\left(\prod_{j=1}^{M_{k}} T_{k}^{\prec}\left(\nu_{k}, \alpha(j)\right)^{k}\right)^{1 /\left(k M_{k}\right)}
$$

We need to show that the limit on the right-hand side of the above converges to the right-hand side of (4.7). To see this, observe that the limit may be rewritten as

$$
\lim _{k \rightarrow \infty} \exp \left(\frac{1}{M_{k}} \sum_{j=1}^{M_{k}} \log T_{k}^{\prec}\left(\nu_{k}, \alpha(j)\right)\right) .
$$

We look at the limit of the expression inside the parentheses. If we fix a compact convex body $Q \subset \operatorname{int}(C)$, then

$$
\lim _{k \rightarrow \infty} \frac{1}{M_{k}} \sum_{\alpha(j) \in k Q}^{M_{k}} \log T_{k}^{\prec}\left(\nu_{k}, \alpha(j)\right)=\lim _{k \rightarrow \infty} \frac{1}{M_{k}} \sum_{\alpha(j) \in k Q} \log T_{\prec}^{w}\left(K, \theta_{j}\right)
$$


where $\theta_{j}=\alpha(j) / k$, since for fixed $k$ the difference between the quantities on each side is bounded above by

$$
\sup \left\{|\log | T_{k}^{\prec}\left(\nu_{k}, \alpha(j)\right)-\log \left|T_{\prec}^{w}\left(K, \theta_{j}\right)\right|: j \in\left\{1, \ldots, N_{k}\right\}\right\},
$$

and this goes to zero by Corollary 2.11 (to Lemma 2.10).

Since $\left\{\theta_{j}: j=1, \ldots, M_{k}\right\}=\frac{1}{k} \mathbb{Z}^{N} \cap C$, the discrete measure $\frac{1}{M_{k}} \sum_{j=1}^{M_{k}} \delta_{\theta_{j}}$, supported on a uniform grid, clearly converges weak-* on $\operatorname{int}(C)$ to the uniform measure $\frac{1}{\operatorname{vol}_{N}(C)} d \theta$ as $k \rightarrow \infty$. Hence

$$
\lim _{k \rightarrow \infty} \frac{1}{M_{k}} \sum_{\theta_{j} \in Q} \log T_{\prec}^{w}\left(K, \theta_{j}\right)=\frac{1}{\operatorname{vol}_{N}(C)} \int_{Q} \log T_{\prec}^{w}(K, \theta) d \theta
$$

Since $Q$ was arbitrary, one can consider a sequence of compact convex sets increasing to $\operatorname{int}(C)$ and obtain

$$
\lim _{k \rightarrow \infty} \frac{1}{M_{k}} \sum_{j=1}^{M_{k}} \log T_{\prec}^{w}\left(K, \theta_{j}\right)=\frac{1}{\operatorname{vol}_{N}(C)} \int_{\operatorname{int}(C)} \log T_{\prec}^{w}(K, \theta) d \theta,
$$

by a dominated convergence argument. 4.2

The formula for transfinite diameter follows by applying the third limit in Lemma

We have a corresponding integral formula in terms of $T_{C}^{w}(K, \theta)$.

Theorem 4.7. We have

$$
D^{w}(K)=\exp \left(\frac{1}{\operatorname{vol}_{N}(C)} \int_{\operatorname{int}(C)} \log T_{C}^{w}(K, \theta) d \theta\right) .
$$

Proof. By Lemmas 4.4 and 4.5 we have

$$
\frac{1}{M_{k} !} V_{k}^{w}\left(K, M_{k}\right) \leq \prod_{\alpha \in k C} T_{k}^{C}\left(\nu_{k}, \alpha\right)^{k} \leq V_{k}^{w}\left(K, M_{k}\right) \quad \text { for any } k \in \mathbb{N}
$$

and taking logs we obtain

$$
-\frac{\log \left(M_{k} !\right)}{k M_{k}}+\log V_{k}^{w}\left(K, M_{k}\right)^{\frac{1}{k M_{k}}} \leq \frac{1}{M_{k}} \sum_{\alpha \in k C} \log T_{k}^{C}\left(\nu_{k}, \alpha\right) \leq \log V_{k}^{w}\left(K, M_{k}\right)^{\frac{1}{k M_{k}}} .
$$

We will apply measure theory to this estimate. But first we will take care of some technicalities before returning to the proof.

Introduce the following notation. Given $\alpha \in \mathbb{Z}^{N}$, let

$$
E_{\alpha, k}:=\left\{\theta \in \mathbb{R}^{N}:|\theta-\alpha / k|<|\theta-\beta / k| \text { for any } \beta \in \mathbb{Z}^{N}\right\},
$$

and let $\bar{E}_{\alpha, k}$ be its closure. By elementary geometry, $E_{\alpha, k}$ is an open $N$ dimensional cube centered at $\alpha / k$, with side length $1 / k$ and faces parallel to the coordinate hyperplanes. The collection $\left\{E_{\alpha, k}\right\}_{\alpha \in \mathbb{Z}^{N}}$ forms a grid in $\mathbb{R}^{N}$.

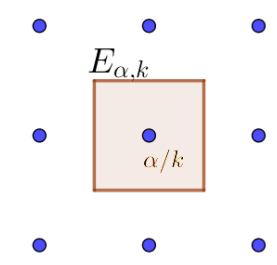


Define the following subsets of $\mathbb{Z}^{N}$ :

$$
\begin{aligned}
\mathcal{N}_{k} & :=\left\{\alpha \in \mathbb{Z}^{N}: \alpha \in k C\right\}, \\
\overline{\mathcal{N}}_{k} & :=\left\{\alpha \in \mathbb{Z}^{N}: \bar{E}_{\alpha, k} \cap C \neq \emptyset\right\}, \\
\partial \mathcal{N}_{k} & :=\left\{\alpha \in \mathbb{Z}^{N}: \bar{E}_{\alpha, k} \cap \partial C \neq \emptyset\right\}, \\
\mathcal{N}_{k}^{\circ} & :=\overline{\mathcal{N}}_{k} \backslash \partial \mathcal{N}_{k} .
\end{aligned}
$$

By the notation defined earlier, $M_{k}$ is the size of $\mathcal{N}_{k}$. We will denote by $\bar{M}_{k}, B_{k}$ and $M_{k}^{\circ}$ the sizes of the other three sets, respectively. (So $M_{k}^{\circ}=\bar{M}_{k}-B_{k}$.)

Lemma 4.8. We have

$$
\mathcal{N}_{k}^{\circ} \subseteq\left\{\alpha \in \mathbb{Z}^{N}: E_{\alpha, k} \subseteq \operatorname{int}(C)\right\} \quad \text { and } \mathcal{N}_{k}^{\circ} \subseteq \mathcal{N}_{k} \subseteq \overline{\mathcal{N}}_{k}
$$

Also, $\frac{B_{k}}{M_{k}}=O\left(\frac{1}{k}\right)$, so that $\frac{B_{k}}{M_{k}}, \frac{B_{k}}{M_{k}^{\circ}} \rightarrow 0$ and $\frac{\bar{M}_{k}}{M_{k}}, \frac{M_{k}^{\circ}}{M_{k}} \rightarrow 1$ as $k \rightarrow \infty$.

Proof. If $\alpha \in k C$ then $E_{\alpha, k} \cap C \neq \emptyset$, so $\mathcal{N}_{k} \subseteq \overline{\mathcal{N}}_{k}$ follows. If $\alpha \in \mathcal{N}_{k}^{\circ}$, then $\bar{E}_{\alpha, k} \cap \partial C=\emptyset$. It follows, since $C$ and $\bar{E}_{\alpha, k}$ are connected, that $E_{\alpha, k} \subseteq \operatorname{int}(C)$. This also implies that $\alpha \in \operatorname{int}(k C)$, so $\mathcal{N}_{k}^{\circ} \subseteq \mathcal{N}_{k}$. The first statement is proved.

By construction, $\left\{\bar{E}_{\alpha}\right\}_{\alpha \in \overline{\mathcal{N}}_{k}}$ and $\left\{\bar{E}_{\alpha}\right\}_{\alpha \in \partial \mathcal{N}_{k}}$ are precisely those cubes that cover $C$ and $\partial C$ respectively. We claim that $M_{k}=O\left(k^{N}\right)$ and $B_{k}=O\left(k^{N-1}\right)$, which is the same as saying that the box-counting dimensions of $C, \partial C$ are $N, N-1$ respectively. This is true because $C$ is a convex set in $\mathbb{R}^{N}$ with nonempty interior Then $M_{k}=O\left(k^{N}\right)$ and $B_{k}=O\left(k^{N-1}\right)$, and the second statement follows immediately.

Define the function $\ell_{k}: C \rightarrow[0, \infty)$ by

$$
\ell_{k}(\theta):=\sum_{\alpha \in \mathcal{N}_{k}} \log T_{k}^{C}\left(\nu_{k}, \alpha\right) \chi_{E_{\alpha, k}}(\theta)
$$

where $\chi_{E_{\alpha, k}}$ denotes the characteristic function of $E_{\alpha, k}$. So $\ell_{k}(\theta)=T_{k}^{C}\left(\nu_{k}, \alpha\right)$ for $\theta \in E_{\alpha, k}$; in particular, when $|\theta-\alpha / k| \leq \frac{N}{2 k}$. Together with Lemma 3.3. we have

$$
\ell_{k}(\theta) \rightarrow \log T_{C}^{w}(K, \theta) \text { pointwise, as } k \rightarrow \infty
$$

Now

$$
\int_{\operatorname{int}(C)} \ell_{k}(\theta) d \theta=\sum_{\alpha \in \mathcal{N}_{k}} \log T_{k}^{C}\left(\nu_{k}, \alpha\right) \operatorname{vol}_{N}\left(E_{\alpha, k} \cap C\right),
$$

so by the first statement of Lemma 4.8 , we have the bounds

$$
\sum_{\alpha \in \mathcal{N}_{k}^{\circ}} \log T_{k}^{C}\left(\nu_{k}, \alpha\right) \operatorname{vol}_{N}\left(E_{\alpha, k}\right) \leq \int_{\operatorname{int}(C)} \ell_{k}(\theta) d \theta \leq \sum_{\alpha \in \overline{\mathcal{N}}_{k}} \log T_{k}^{C}\left(\nu_{k}, \alpha\right) \operatorname{vol}_{N}\left(E_{\alpha, k}\right) .
$$

Similarly,

$$
\frac{M_{k}^{\circ}}{k^{N}}=\sum_{\alpha \in \mathcal{N}_{k}^{\circ}} \operatorname{vol}_{N}\left(E_{\alpha, k}\right) \leq \operatorname{vol}_{N}(C) \leq \sum_{\alpha \in \overline{\mathcal{N}}_{k}} \operatorname{vol}_{N}\left(E_{\alpha, k}\right)=\frac{\bar{M}_{k}}{k^{N}}
$$

Lemma 4.9. We have

$$
\left|\frac{1}{\operatorname{vol}_{N}(C)} \int_{\operatorname{int}(C)} \ell_{k}(\theta) d \theta-\frac{1}{M_{k}} \sum_{\alpha \in \mathcal{N}_{k}} \log T_{k}^{C}\left(\nu_{k}, \alpha\right)\right| \rightarrow 0 \text { as } k \rightarrow \infty .
$$

IIn particular, $\partial C$ is locally the graph of a Lipschitz map, so the box-counting dimension is the same as that of an $(N-1)$-dimensional hyperplane (i.e., $N-1)$. 
Proof. We will prove the lemma by showing that

$$
\limsup _{k \rightarrow \infty}\left(\frac{1}{\operatorname{vol}_{N}(C)} \int_{\operatorname{int}(C)} \ell_{k}(\theta) d \theta-\frac{1}{M_{k}} \sum_{\alpha \in \mathcal{N}_{k}} \log T_{k}^{C}\left(\nu_{k}, \alpha\right)\right) \leq 0
$$

and

$$
\liminf _{k \rightarrow \infty}\left(\frac{1}{\operatorname{vol}_{N}(C)} \int_{\operatorname{int}(C)} \ell_{k}(\theta) d \theta-\frac{1}{M_{k}} \sum_{\alpha \in \mathcal{N}_{k}} \log T_{k}^{C}\left(\nu_{k}, \alpha\right)\right) \geq 0 .
$$

By 4.13, 4.14 and the fact that $\operatorname{vol}_{N}\left(E_{\alpha, k}\right)=\left(\frac{1}{k}\right)^{N}$ for all $\alpha$,

$$
\begin{aligned}
\frac{1}{\operatorname{vol}_{N}(C)} \int_{\operatorname{int}(C)} l_{k}(\theta) d \theta & \leq \frac{k^{N}}{M_{k}^{\circ}} \sum_{\alpha \in \overline{\mathcal{N}}_{k}} \log T_{k}^{C}\left(\nu_{k}, \alpha\right)\left(\frac{1}{k}\right)^{N} \\
& =\frac{1}{M_{k}^{\circ}} \sum_{\alpha \in \overline{\mathcal{N}}_{k}} \log T_{k}^{C}\left(\nu_{k}, \alpha\right) .
\end{aligned}
$$

Hence

$$
\begin{aligned}
& \frac{1}{\operatorname{vol}_{N}(C)} \int_{\operatorname{int}(C)} \ell_{k}(\theta) d \theta-\frac{1}{M_{k}} \sum_{\alpha \in \mathcal{N}_{k}} \log T_{k}^{C}\left(\nu_{k}, \alpha\right) \\
& \quad \leq \frac{1}{M_{k}^{\circ}} \sum_{\alpha \in \overline{\mathcal{N}}_{k}} \log T_{k}^{C}\left(\nu_{k}, \alpha\right)-\frac{1}{M_{k}} \sum_{\alpha \in \mathcal{N}_{k}^{\circ}} \log T_{k}^{C}\left(\nu_{k}, \alpha\right) \\
& \quad=\left(\frac{1}{M_{k}^{\circ}}-\frac{1}{M_{k}}\right) \sum_{\alpha \in \mathcal{N}_{k}^{\circ}} \log T_{k}^{C}\left(\nu_{k}, \alpha\right)+\frac{1}{M_{k}^{\circ}} \sum_{\alpha \in \partial \mathcal{N}_{k}} \log T_{k}^{C}\left(\nu_{k}, \alpha\right) \\
& \leq\left(\frac{1}{M_{k}^{\circ}}-\frac{1}{M_{k}}\right) M_{k}^{\circ} \log R+\frac{1}{M_{k}^{\circ}} B_{k} \log R \\
& \quad=\left(1-\frac{M_{k}^{\circ}}{M_{k}}+\frac{B_{k}}{M_{k}^{\circ}}\right) \log R \longrightarrow 0 \text { as } k \rightarrow 0
\end{aligned}
$$

by Lemma 4.8. Hence we obtain 4.15. Next, using (4.13), 4.14 to estimate from below, we obtain by a similar calculation that

$$
\begin{aligned}
\frac{1}{\operatorname{vol}_{N}(C)} \int_{\operatorname{int}(C)} \ell_{k}(\theta) d \theta-\frac{1}{M_{k}} \sum_{\alpha \in \mathcal{N}_{k}} \log T_{k}^{C}\left(\nu_{k}, \alpha\right) & \geq\left(\frac{M_{k}^{\circ}}{\overline{M_{k}}}-\frac{M_{k}^{\circ}}{M_{k}}-\frac{B_{k}}{M_{k}}\right) \log R \\
& \longrightarrow 0 \quad \text { as } k \rightarrow 0,
\end{aligned}
$$

which proves 4.16.

End of the proof of Theorem 4.7. By the estimate 4.10 and Theorem 4.6.

$$
\lim _{k \rightarrow \infty}\left(\frac{1}{M_{k}} \sum_{\alpha \in \mathcal{N}_{k}} \log T_{k}^{C}\left(\nu_{k}, \alpha\right)\right)=\log D^{w}(K) .
$$

Applying Lemma 4.9

$$
\lim _{k \rightarrow \infty}\left(\frac{1}{\operatorname{vol}_{N}(C)} \int_{\operatorname{int}(C)} \ell_{k}(\theta) d \theta\right)=\log D^{w}(K) .
$$

Finally, using 4.12 and the dominated convergence theorem, the theorem follows. 


\section{C-LEJA POINTS}

To define $C$-Leja points, (called Leja points from now on, for convenience) we use the modified grevlex ordering $\prec_{C}$; enumerate the monomials $\left\{z^{\alpha(j)}\right\}_{j=1}^{\infty}$ in increasing order according to $\prec_{C}$. We will also write

$$
\operatorname{VDM}\left(\zeta_{1}, \ldots, \zeta_{j}\right):=\operatorname{VD} M_{k}^{w}\left(\zeta_{1}, \ldots, \zeta_{j}\right)
$$

where $k=k(j):=\operatorname{deg}_{C}\left(z^{\alpha(j)}\right)$ and $w$ is a fixed weight function on $K$.

A sequence of Leja points for $K$ is a sequence

$$
\mathcal{L}:=\left\{\zeta_{j}\right\}_{j=1}^{\infty}=\bigcup_{s \in \mathbb{N}} \mathcal{L}_{s}
$$

where $\mathcal{L}_{s}=\left\{\zeta_{j}\right\}_{j=1}^{s}$ is constructed inductively: first set $\mathcal{L}_{1}:=\left\{\zeta_{1}\right\}$ for some $\zeta_{1} \in K$ for which $w\left(\zeta_{1}\right) \neq 0$; then given $\mathcal{L}_{s}$, form $\mathcal{L}_{s+1}:=\mathcal{L}_{s} \cup\left\{\zeta_{s+1}\right\}$ by picking $\zeta_{s+1} \in K$ that satisfies

$$
\left|V D M\left(\zeta_{1}, \ldots, \zeta_{s}, \zeta_{s+1}\right)\right|=\sup _{\eta \in K}\left|V D M\left(\zeta_{1}, \ldots, \zeta_{s}, \eta\right)\right|
$$

The Leja points of order $k$ are those of the set $\mathcal{L}_{M_{k}}$.

Assume condition $(\dagger)$ of Section 3 holds for $C$. We now show that in the unweighted case $(w \equiv 1)$, Leja points give the transfinite diameter $\delta_{C}(K)$. The statement for weighted Leja points is an open conjecture, even in the classical case $C=\Sigma$.

Theorem 5.1. Let $\left\{\zeta_{j}\right\}_{j=1}^{\infty}$ be a sequence of (unweighted) Leja points for $K$, and let $L_{s}:=\operatorname{VDM}\left(\zeta_{1}, \ldots, \zeta_{s}\right)$. Then

$$
\lim _{k \rightarrow \infty}\left(L_{M_{k}}\right)^{1 / L_{k}}=\delta_{C}(K) .
$$

Before beginning the proof, note that by definition, $L_{M_{k}} \leq V_{M_{k}}$, so $\delta_{C}(K)$ is an upper bound for the lim sup of the left-hand side as $k \rightarrow \infty$. To get a lower bound for the lim inf we will bound $L_{M_{k}}$ from below. The argument is similar to that of Proposition 3.7 in [5]. First we need a lemma comparing Chebyshev constants.

Lemma 5.2. Let $\alpha \in \mathbb{Z}^{N}$ and $j, k \in \mathbb{N}$ with $\alpha \in j C \subset k C$. Then

$$
T_{k}^{C}\left(\nu_{k}, \alpha\right)^{k}=T_{j}^{C}\left(\nu_{j}, \alpha\right)^{j} .
$$

Proof. By the definition of $\prec_{C}$, we have $\mathcal{M}_{j}^{C}(\alpha)=\mathcal{M}_{k}^{C}(\alpha)$, as all lower terms for the latter class are in $j C$. Since the classes are the same, the Chebyshev constants are the same: if $p$ is a polynomial in the class that maximizes $\|p\|_{K}$, then $T_{k}^{C}\left(\nu_{k}, \alpha\right)^{k}=$ $\|p\|_{K}=T_{j}^{C}\left(\nu_{j}, \alpha\right)^{j}$.

Remark 5.3. The above proof only works in the unweighted setting. If $w \neq \equiv 1$ and $j \neq k$ then $\left\|w^{k} p\right\|_{K}$ and $\left\|w^{j} p\right\|_{K}$ are not necessarily equal.

Lemma 5.4. Let $s \in \mathbb{N}$. Then $L_{s} \geq T_{k}^{C}(\alpha(s))^{k} L_{s-1}$.

Proof. By the definition of $L_{s}$, the point $\zeta_{s} \in K$ maximizes $\operatorname{VDM}\left(\zeta_{1}, \ldots, \zeta_{s-1}, \eta\right)$ over all $\eta \in K$, which by the proof of Lemma 4.5. maximizes

$$
\left|V D M\left(\zeta_{1}, \ldots, \zeta_{s-1}\right)\right| \cdot|p(\eta)|
$$

over all $\eta \in K$ for some polynomial $p \in \mathcal{M}_{k}^{C}(\alpha(s))$. Hence

$$
\begin{aligned}
L_{s} & =\left|\operatorname{VDM}\left(\zeta_{1}, \ldots, \zeta_{s}\right)\right| \\
& =\left|p\left(\zeta_{s}\right)\right| \cdot\left|\operatorname{VDM}\left(\zeta_{1}, \ldots, \zeta_{s-1}\right)\right| \\
& =\|p\|_{K}\left|\operatorname{VDM}\left(\zeta_{1}, \ldots, \zeta_{s-1}\right)\right|=\|p\|_{K} L_{s-1} \geq T_{k}^{C}(\alpha(s))^{k} L_{s-1} .
\end{aligned}
$$


Proof of Theorem 5.1. Let $k \in \mathbb{N}$. By repeatedly applying the previous lemma for all indices in $k C \backslash(k-1) C$, we obtain

$$
L_{M_{k}} \geq L_{M_{k-1}} \prod_{s=M_{k-1}+1}^{M_{k}} T_{k}^{C}(\alpha(s))^{k} .
$$

Iterating the above estimate another $k-1$ times, we obtain

$$
L_{M_{k}} \geq \prod_{j=0}^{k}\left(\prod_{s=M_{j-1}+1}^{M_{j}} T_{j}^{C}(\alpha(s))^{j}\right)=\prod_{s=1}^{M_{k}} T_{k}^{C}(\alpha(s))^{k}
$$

where the last equality follows from Lemma 5.2 Putting the right-hand side into the estimate 4.9 , we obtain

$$
L_{M_{k}} \geq \frac{1}{M_{k} !} V_{k}\left(K, M_{k}\right) .
$$

Taking $L_{k}$-th roots and letting $k \rightarrow \infty$, the result follows by Theorem 4.6

Remark 5.5. Let $\mu_{k}:=\frac{1}{M_{k}} \sum_{j=1}^{M_{k}} \delta_{\zeta_{j}}$ be the discrete probability measure at the Leja points of order $k$. Putting together Theorem 5.1 and Corollary 6.5 from [2], we have weak-* convergence to the equilibrium measure:

$$
\mu_{k} \rightarrow\left(d d^{c} V_{C, K}\right)^{N} \text { as } k \rightarrow \infty .
$$

Acknowledgement. I would like to thank Norm Levenberg for pointing out an error in an earlier draft of the paper.

\section{ReFERENCES}

[1] Turgay Bayraktar. Zero distribution of random sparse polynomials. Michigan Math. J., $66: 389-419,2017$.

[2] Turgay Bayraktar, Thomas Bloom, and Norm Levenberg. Pluripotential theory and convex bodies. Mat. Sb., 209:352-384, 2018.

[3] Robert Berman and Sébastien Boucksom. Growth of balls of holomorphic sections and energy at equilibrium. Invent. Math., 181:337-394, 2010.

[4] Robert Berman, Sébastien Boucksom, and David Witt Nyström. Fekete points and convergence towards equilibrium measures on complex manifolds. Acta. Math., 207(1):1-27, 2011.

[5] Thomas Bloom, Len Bos, C. Christensen, and Norman Levenberg. Polynomial interpolation of holomorphic functions in $\mathbb{C}$ and $\mathbb{C}^{n}$. Rocky Mountain J. Math, 22(2):441-470, 1992.

[6] Thomas Bloom and Norman Levenberg. Weighted pluripotential theory in $\mathbb{C}^{n}$. Amer. J. Math., 125(1):57-103, 2003.

[7] Len Bos and Norm Levenberg. Bernstein-Walsh theory associated to convex bodies and applications to multivariate approximation theory. Comput. Methods Funct. Theory, 18:361-388, 2018.

[8] Sione Ma'u. Newton-Okounkov bodies and transfinite diameter. Dolomites Res. Notes Approx., 10(Special Issue):138-160, 2017.

[9] Lloyd N. Trefethen. Multivariate polynomial approximation in the hypercube. Proc. Amer. Math. Soc., 145(11):4837-4844, 2017.

[10] David Witt Nyström. Transforming metrics on a line bundle to the Okounkov body. Ann. Sci. Éc. Norm. Supér., 47(4):1111-1161, 2014.

[11] V.P. Zaharjuta. Transfinite diameter, Chebyshev constants, and capacity for compacta in $\mathbb{C}^{n}$. Math. USSR Sbornik, 25(3):350-364, 1975.

Department of Mathematics, University of Auckland, Auckland, NZ

E-mail address: s.mau@auckland.ac.nz 